\title{
Prognostic Impact of Microsatellite Instability in Colorectal Cancer Presenting With Mucinous, Signet-Ring, and Poorly Differentiated Cells
}

\author{
Sang Hun Jung, So Hyun Kim, Jae Hwang Kim \\ Department of Surgery, Yeungnam University College of Medicine, Daegu, Korea
}

Purpose: Mucinous cells (MUCs), signet-ring cells (SRCs), and poorly differentiated cells (PDCs) are uncommon histologic types and have been associated with advanced tumor stage and poor prognosis. However, MUCs, SRCs, and PDCs are commonly observed in cancers with high microsatellite instability (MSI), which have favorable outcomes compared with cancers with microsatellite stability (MSS). The purpose of this study was to evaluate the prognostic impact of highMSI in patients with sporadic colorectal cancer presenting with MUCs, SRCs, and/or PDCs.

Methods: Between January 2006 and December 2012, 176 with proven microsatellite status who also presented with MUCs, SRCs, and PDCs were selected for this study and were divided into 2 groups, high-MSI and MSS; their outcomes were analyzed.

Results: Of the 176 patients, 56 and 120, respectively, had high-MSI and MSS cancers. High-MSI cancers had larger tumors, proximal tumor location, and a lower TNM stage. The recurrence rate was lower in the high-MSI group (13.7\% vs. $35.4 \%, \mathrm{P}=0.006)$. Common patterns of distant metastasis for MUC, SRC, PDC cancers were peritoneal spread $(46.9 \%)$ and hematogenous metastasis (46.4\%). The 5-year CSS rates were $88.2 \%$ and $61.2 \%$ for patients with high-MSI and MSS cancers, respectively $(\mathrm{P}<0.0001)$. In the multivariate analysis, except for stage-IV cancer, MSI status was an independent risk factor for cancer-specific survival (MSS: hazard ratio, 4.34; 95\% confidence interval, 1.68-11.21).

Conclusion: In patients with colorectal cancer presenting with MUCs, SRCs, and/or PDCs, those with high-MSI cancers had better outcomes.

Keywords: Microsatellite instability; Mucinous cells; Signet-ring cells; Poorly differentiated cells; Colorectal neoplasms

\section{INTRODUCTION}

Colorectal cancer (CRC) is one of the most common cancers worldwide, and several histological types of adenocarcinomas

Received: October 1,2015 - Accepted: March 2, 2016

Correspondence to: So Hyun Kim, M.D.

Department of Surgery, Yeungnam University Medical Center,

170 Hyeonchung-ro, Nam-gu, Daegu 42415, Korea

Tel:+82-53-620-3580, Fax: +82-53-624-1213

E-mail: coexist21@hanmail.net

Main topic was presented at the Annual Congress of the Korean Surgical Society, 2014. Seoul, Korea

(c) 2016 The Korean Society of Coloproctology

This is an open-access article distributed under the terms of the Creative Commons Attribution NonCommercial License (http://creativecommons.org/licenses/by-nc/4.0) which permits unrestricted noncommercial use, distribution, and reproduction in any medium, provided the original work is properly cited. have been reported to be associated with varied survival outcomes [1-4]. Mucinous cell (MUC), signet-ring cell (SRC), and poorly differentiated cell (PDC) cancers are uncommon histologic types, with MUCs being present in $5 \%-12 \%$ of CRCs, SRCs in $0 \%-1 \%$, and PDCs in 5\%-20\% [5, 6]. However, these histologic types are generally associated with an advanced tumor stage and poor patient outcomes [1-3, 7]. Although CRCs presenting with MUCs, SRCs, and PDCs are histologically inhomogenous, they are reported to share similar clinicopathologic features and biologic behaviors $[8,9]$. Especially, the distinct clinical and pathologic features among these histologic types mainly depend on the microsatellite instability (MSI) status [10], with MUCs, SRCs, and PDCs being more frequently seen in high-MSI tumors than in microsatellite stable (MSS) tumors $[8,11]$.

MSI, which represents a defective mismatch repair gene, has been identified in most cancers in individuals with hereditary 
non-polyposis CRC; however, the majority of MSI tumors are found in individuals with sporadic disease [12]. About $15 \%$ of all colorectal carcinomas have MSI, and $75 \%-80 \%$ of that group has acquired methylation of $M L H 1$; only $2 \%-3 \%$ of all CRCs have germline mutations in one of the mismatch repair genes [12]. CRCs presenting with MUCs, SRCs, and PDCs show more aggressive pathologic features and poorer prognosis than those presenting with well- or moderately differentiated cells; however, MSI cancers, which include many of these aggressive histologic types, show a relatively favorable prognosis compared with MSS cancers [11]. However, data on the MSI status as a prognostic factor for CRC remains inconclusive because of the small numbers of patients and the different histologies in the studies $[2,11]$. The present study aimed to evaluate the prognostic impact of MSI in patients with sporadic CRC presenting with mucinous, signetring, and poorly differentiated adenocarcinomas.

\section{METHODS}

This is a retrospective study of 1,232 CRC patients with proven microsatellite status from 2006 to 2012 . Of those, 176 patients presented with MUCs, SRCs, and PDCs and with proven microsatellite status in cancer tissue after resection of the primary CRC. Patients who presented with metachronous or recurrent cancer, inflammatory bowel disease, familial adenomatous polyposis, and positive family history suggestive of hereditary nonpolyposis CRC by the Amsterdam I \& II criteria were excluded.

Classifications of MUCs, SRCs, and PDCs were performed in accordance with the World Health Organization (WHO) criteria: a mucinous carcinoma with its extracellular mucinous component representing more than $50 \%$ of the lesion; a SRC carcinoma with the presence of SRCs (intracytoplasmic mucin) in more than $50 \%$ of the lesion; a poorly differentiated carcinoma with less than $50 \%$ of the lesion showing poor gland development with the formation of irregular clusters or sheets [11]. All tumors were staged according to the sixth edition of the TNM classification of American Joint Committee on Cancer. The sites of the primary cancers were divided into right colon (from the cecum to the distal transverse colon) and left colon or rectum (from the splenic flexure to the rectum). Distant metastatic sites were stratified into three main spread patterns: hematogenous (liver, lungs, bones), lymphatic (systemic lymph node, lymphangitic pulmonary metastasis) and peritoneum (peritoneum/ovary).

Molecular analysis for MSI status was performed using a standard polymerase chain reaction (PCR) method [12]. Tissue samples from the tumor and the normal colonic mucosa were obtained from each patient after resection. After DNA isolation from a paraffin-embedded specimen from each patient, an MSI analysis was performed with the five microsatellite markers of the Bethesda Consensus Panel (D2S123, D17S250, D5S346, BAT25, and BAT26). MSI was determined by using the mobility shifts of the products from PCR. In tumors with MSI, additional bands were found in the normal allele regions. As the National Cancer Institute recommends, tumors with instability at two or more microsatellite loci were defined as high-MSI tumors; those with instability at one marker, as low-frequency microsatellite unstable (low-MSI); and those without instability, as MSS [12].

The clinicopathological data, including age (years), sex, initial carcinoembryonic antigen (CEA) value $(\mathrm{ng} / \mathrm{mL})$, tumor location (right versus left), tumor size $(\mathrm{cm})$, lymphatic invasion, vascular invasion, neural invasion, TNM classification, MSI status, site of distant metastasis, and postoperative survival, were analyzed. The median follow-up period of the study was 38 months. We divided the 176 patients into 2 subgroups: patients who had high-MSI and those who had MSS (and low-MSI) $[8,12]$. Patients were followed up at 3-month intervals for the first 2 years and then at 6-month intervals for the last 3 years to evaluate recurrence and at 2 - to 3-month intervals to assess disease progression. Follow-up examinations included physical examinations, determinations of CEA level, and computed tomography or positron emission tomography scans. Data were described as categorical variables by using the Pearson chi-square test or Fischer exact test. The Kaplan-Meier method with the log-rank test was used to conduct the survival analysis according to MSI status. The cancer-specific survival (CSS) was computed from the date of surgery to the date when the patient was last known to be alive. Finally, a multivariate Cox regression analysis was done to evaluate the independent prognostic factors, adjusting for possible confounding factors. All statistical tests were assessed at the conventional 0.05 level of significance. Statistical analysis was conducted with IBM SPSS ver. 18.0 (IBM Co., Armonk, NY, USA). A P-value of 0.05 or less was considered statistically significant.

\section{RESULTS}

Of the 176 patients, in 90 (51.1\%), the site of the cancer was the right colon, and in 47 of 176 (26.7\%), it was the rectum. The frequencies of high-MSI by cell type were $20 / 70$ for MUC (20 patients with high-MSI/70 patients presenting with MUCs $=28.6 \%$ ), 3 of 15 (20.0\%) for SRC, and 33 of 91 (36.3\%) for PDC. The clinicopathologic characteristics according to MSI status are summarized in Table 1. The 176 included 101 males (57.4\%) and $75 \mathrm{fe}-$ males $(42.6 \%)$ with a mean age of 59.5 years (range, $17-84$ years). Compared to patients with MSS cancers, those with high-MSI cancers tended to be young ( $\leq 55$ years) $(46.4 \%$ vs. $31.7 \%, \mathrm{P}=$ 0.058). The high-MSI cancer was more commonly located in the right colon than the left colon ( $80.4 \%$ vs. $19.6 \%, \mathrm{P}<0.001)$. HighMSI cancers had more favorable pathologic features than MSS tumors (vascular invasion: $10.7 \%$ vs. $25.8 \%, \mathrm{P}=0.002$; neural invasion: $25.0 \%$ vs. $52.5 \%, \mathrm{P}=0.001$ ), even though the size of highMSI cancers was statistically larger than that of MSS cancers. However, the frequencies of lymphatic invasion in patients with high-MSI and MSS cancers were similar, $67.9 \%$ and $71.7 \%$, respectively $(\mathrm{P}=0.630)$. The incidences of T4 tumors were $25.0 \%$ 
Table 1. Clinicopathologic characteristics according to MSI status in patients with colorectal cancer presenting with MUC/SRC/PDC $(\mathrm{n}=176)$

\begin{tabular}{|c|c|c|c|}
\hline Characteristic & MSS/low $(n=120)$ & High-MSI $(n=56)$ & P-value \\
\hline Sex & & & 0.206 \\
\hline Male & $65(54.2)$ & $36(64.3)$ & \\
\hline Female & $55(45.8)$ & 20 (35.7) & \\
\hline Age (yr) & & & 0.058 \\
\hline$\leq 55$ & $38(31.7)$ & $26(46.4)$ & \\
\hline$>55$ & $82(68.3)$ & 30 (53.6) & \\
\hline Initial CEA (ng/mL) & & & 0.283 \\
\hline$\leq 5$ & $56(46.7)$ & $31(55.4)$ & \\
\hline$>5$ & $64(53.3)$ & 25 (44.6) & \\
\hline Tumor location & & & $<0.001$ \\
\hline Right & $45(37.5)$ & $45(80.4)$ & \\
\hline Left $^{\mathrm{a}}$ & 75 (62.5) & $11(19.6)$ & \\
\hline Cell types & & & 0.344 \\
\hline PDC (36.3) & $58(48.3)$ & $33(58.9)$ & \\
\hline MUC (28.6) & $50(41.7)$ & 20 (35.7) & \\
\hline $\operatorname{SRC}(20.0)$ & $12(10.0)$ & $3(5.4)$ & \\
\hline Tumor size (cm) & & & 0.003 \\
\hline$\leq 6$ & 76 (63.3) & 22 (39.3) & \\
\hline$>6$ & $44(36.7)$ & $34(60.7)$ & \\
\hline Lymphatic invasion & & & 0.606 \\
\hline No & $34(28.3)$ & $18(32.1)$ & \\
\hline Yes & $86(71.7)$ & $38(67.9)$ & \\
\hline Vascular invasion & & & 0.002 \\
\hline No & 89 (74.2) & 50 (89.3) & \\
\hline Yes & $31(25.8)$ & $6(10.7)$ & \\
\hline Neural invasion & & & 0.001 \\
\hline No & $57(47.5)$ & $42(75.0)$ & \\
\hline Yes & $63(52.5)$ & $14(25.0)$ & \\
\hline Depth of invasion & & & 0.080 \\
\hline $\mathrm{T} 1 / 2$ & $6(5.0)$ & $4(7.1)$ & \\
\hline T3 & $63(52.5)$ & $38(67.9)$ & \\
\hline $\mathrm{T} 4$ & $51(42.5)$ & $14(25.0)$ & \\
\hline Lymph-node metastasis & & & $<0.001$ \\
\hline NO & 39 (32.5) & $32(57.1)$ & \\
\hline $\mathrm{N} 1$ & $21(17.5)$ & 17 (30.4) & \\
\hline N2 & $60(50.0)$ & 7 (12.5) & \\
\hline TNM stage & & & 0.001 \\
\hline 1 & $7(5.8)$ & $3(5.4)$ & \\
\hline$\|$ & $31(25.8)$ & 29 (51.8) & \\
\hline III & 41 (34.2) & 19 (33.9) & \\
\hline IV & 41 (34.2) & $5(8.9)$ & \\
\hline
\end{tabular}

Values are presented as number (\%).

MSI, microsatellite instability; MUC, mucinous cell; SRC, signet-ring cell; PDC, poorly differentiated cell; MSS, microsatellite stability; CEA, carcinoembryonic antigen.

aLeft tumor location: from splenic flexure to rectum.
Table 2. Systemic metastasis rate and first dissemination organs in patients with colorectal cancer presenting with MUC/SRC/PDC

\begin{tabular}{lcccc}
\hline Variable & $\begin{array}{c}\text { All } \\
(\mathrm{n}=176)\end{array}$ & $\begin{array}{c}\text { MSS/low } \\
(\mathrm{n}=120)\end{array}$ & $\begin{array}{c}\text { High-MSI } \\
(\mathrm{n}=56)\end{array}$ & P-value \\
\hline Overall distant metastasis & $81(46.0)$ & $69(57.5)$ & $12(21.4)$ & $<0.001$ \\
Synchronous (stage IV) & $46(26.1)$ & $41(34.2)$ & $5(8.9)$ & $<0.001$ \\
Metachronous ( $\mathrm{n}=130)^{\mathrm{a}}$ & $35(26.9)$ & $28(35.4)$ & $7(13.7)$ & 0.006 \\
Hematogenous & $37(21.0)$ & $32(26.7)$ & $5(8.9)$ & 0.007 \\
$\quad$ Liver & 24 & 21 & 3 & \\
Lung & 8 & 7 & 1 & \\
$\quad$ Bone & 6 & 5 & 1 & \\
Systemic lymph node & $29(16.5)$ & $23(19.2)$ & $6(10.7)$ & 0.159 \\
Peritoneal seeding/ovary & $38(21.6)$ & $35(29.2)$ & $3(5.4)$ & $<0.001$ \\
\hline
\end{tabular}

Values are presented as number (\%).

MUC, mucinous cell; SRC, signet-ring cell; PDC, poorly differentiated cell; MSS, microsatellite stability; MSI, microsatellite instability.

aStages I//I/III were included.

and $42.5 \%$ in patients with high-MSI and MSS cancers, respectively, but the difference was not significant $(\mathrm{P}=0.080)$. However, the incidence of lymph-node metastasis was significantly lesser in the high-MSI group than in the MSS group ( $42.9 \%$ vs. $67.5 \%, \mathrm{P}<$ 0.001). In addition, the presence of synchronous distant metastasis in patients with high-MSI cancers was lower than it was in patients with MSS cancers $(8.9 \%$ vs. $34.2 \%, \mathrm{P}=0.001)$. The adjuvant chemotherapeutic regimens for stage-II or -III patients $(\mathrm{n}=120)$ were 5-fluorouracil-based $(\mathrm{n}=87)$ and oxaliplatin-based $(\mathrm{n}=37)$ regimens, and 20 patients (11.9\%) had preoperative chemoradiotherapy for locally advanced rectal cancer.

The overall systemic recurrence rate after a resection with curative intent for patients with MUC/SRC/PDC CRCs, except stage IV, was $26.9 \%$ ( 35 of 130 patients). The rate of systemic metastasis for patients with high-MSI cancers was significantly lower than that of patients with MSS cancers (13.7\% [7 of 51 patients] vs. $35.4 \%$ [ 28 of 79 patients], $\mathrm{P}=0.006$ ). The organs of synchronous or metachronous dissemination at first presentation are listed in Table 2. Overall, peritoneal dissemination was present in 38 of 81 patients (46.9\%) with MUC/SRC/PDC CRCs and was common compared with 37 of 80 patients (46.4\%) with hematogenous metastasis regardless of MSI status. The incidence of peritoneal metastasis for patients with high-MSI cancers was significantly less than that of patients with MSS cancers (5.4\% vs. $29.2 \%, \mathrm{P}<$ 0.001 ), and hematogenous metastasis was infrequent in patients with high-MSI cancers compared with those with MSS cancers ( $8.9 \%$ vs. $26.7 \%, P=0.007$ ). However, the incidences of systemic lymph-node metastases in the 2 groups showed no statistically significant difference (10.7\% in the high-MSI group vs. $19.2 \%$ in the MSS group, $\mathrm{P}=0.159$ ).

The overall 5-year cancer-specific survival rates (CSSRs) were $88.2 \%$ and $61.2 \%$ for the high-MSI and the MSS patients, respec- 

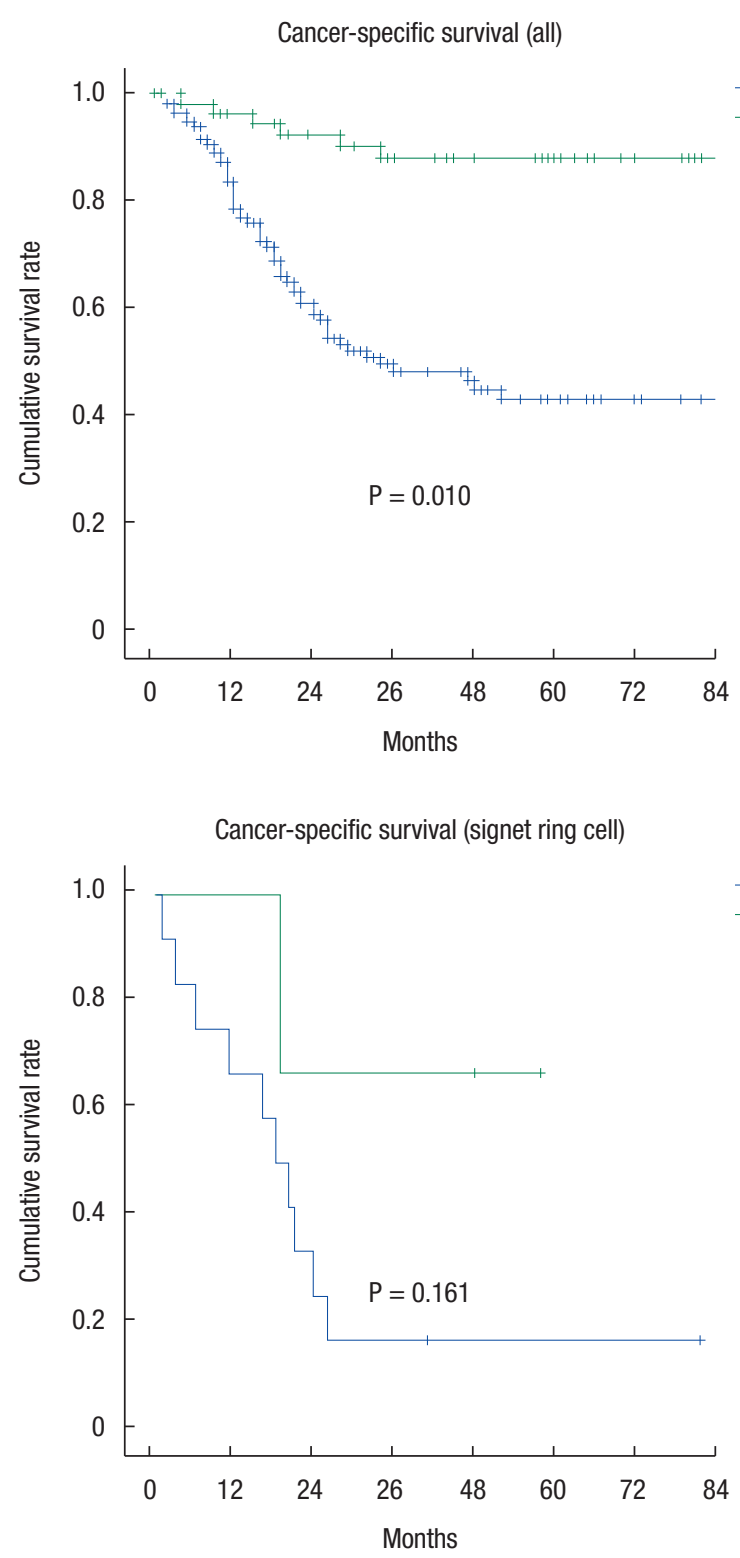
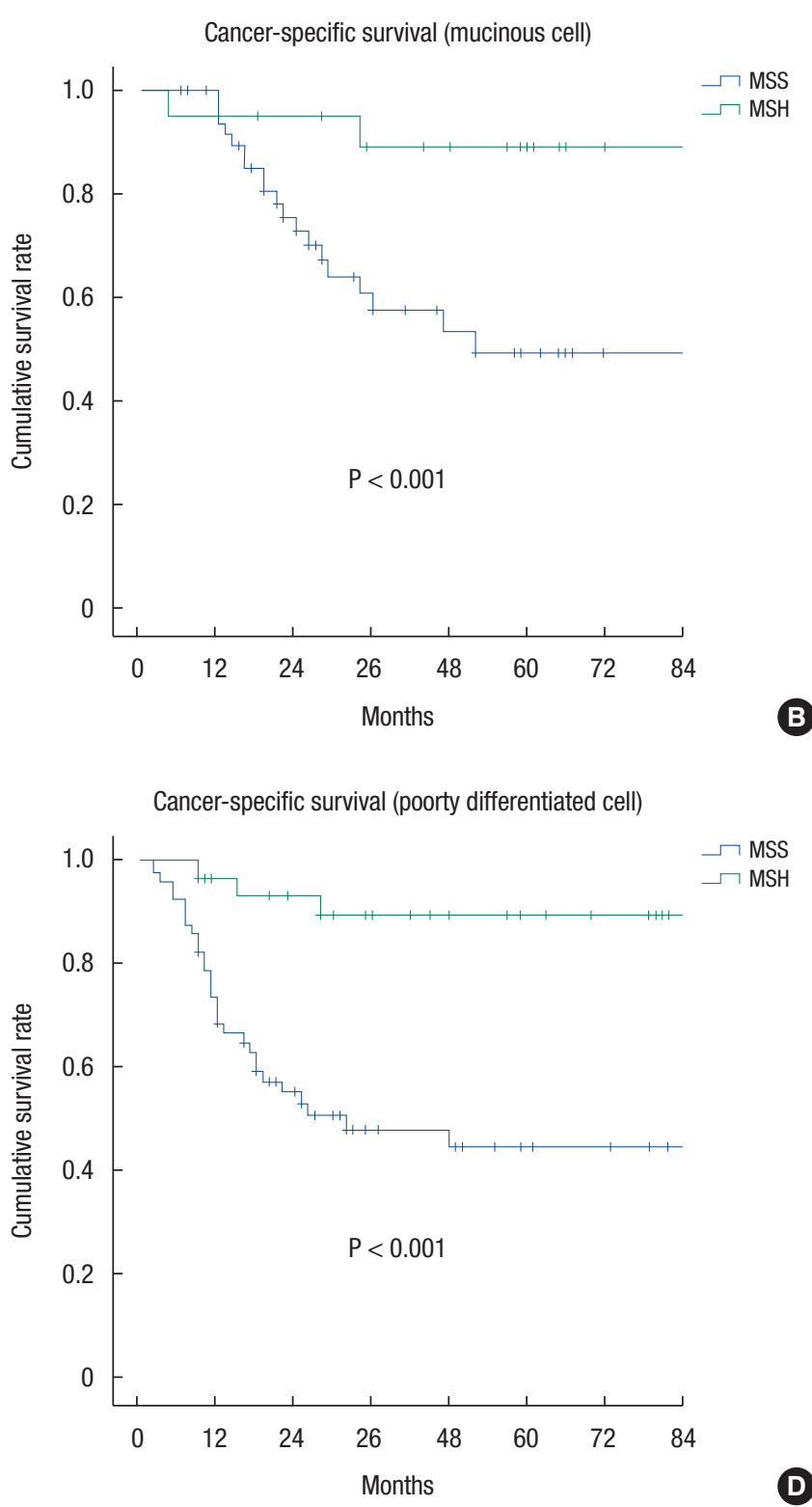

Fig. 1. Kaplan-Meier survival curves showing the 5-year cancer-specific survival rate according to MSI status (cell type). (A) All patients; (B) mucinous cells; (C) signet ring cells; and (D) poorly differentiated cells.

tively $(\mathrm{P}<0.001)$ (Fig. 1). This difference was also demonstrated in the MUC ( $89.1 \%$ vs. $49.6 \%, \mathrm{P}<0.001)$ and the PDC $(89.9 \%$ vs. $44.7 \%, \mathrm{P}<0.001)$ patients, but not in the SRC (66.7\% vs. $16.7 \%, \mathrm{P}$ $=0.161)$ patients. The 5-year CSSR for high-MSI patients, except stage IV, was significantly higher than that of MSS patients $(87.2 \%$ vs. $62.2 \%, \mathrm{P}=0.008$ ) (Fig. 2). In the subgroup analysis, the 5-year CSSR for stage II high-MSI patients was statistically higher than that of MSS patients ( $96.5 \%$ vs. $66.5 \%, \mathrm{P}=0.009)$, but for patients with stage-III cancer, the CSSRs for the two groups were not significantly different ( $71.1 \%$ vs. $55.2 \%, \mathrm{P}=0.161$ ). Of the 5 stage-IV high-MSI patients, 3 underwent a liver resection for curative treatment, and for those 3 patients, no additional recurrences were found during a 5-year follow-up. One high-MSI patient died from a cardiovascular attack, and another underwent radiation therapy for an internal iliac lymph-node metastasis.

To identify the risk factor influencing CSS, we used the Cox regression hazards model to analyze covariates of gender, age, CEA level, tumor location, lymphatic invasion, vascular invasion, neural invasion, T4 invasion, regional lymph-node metastasis, distant metastasis and MSI status. From our analysis, the presence of a MSS tumor was an independent risk factors that worsened CSS compared with the presence of a high-MSI tumor (CSS: hazard 

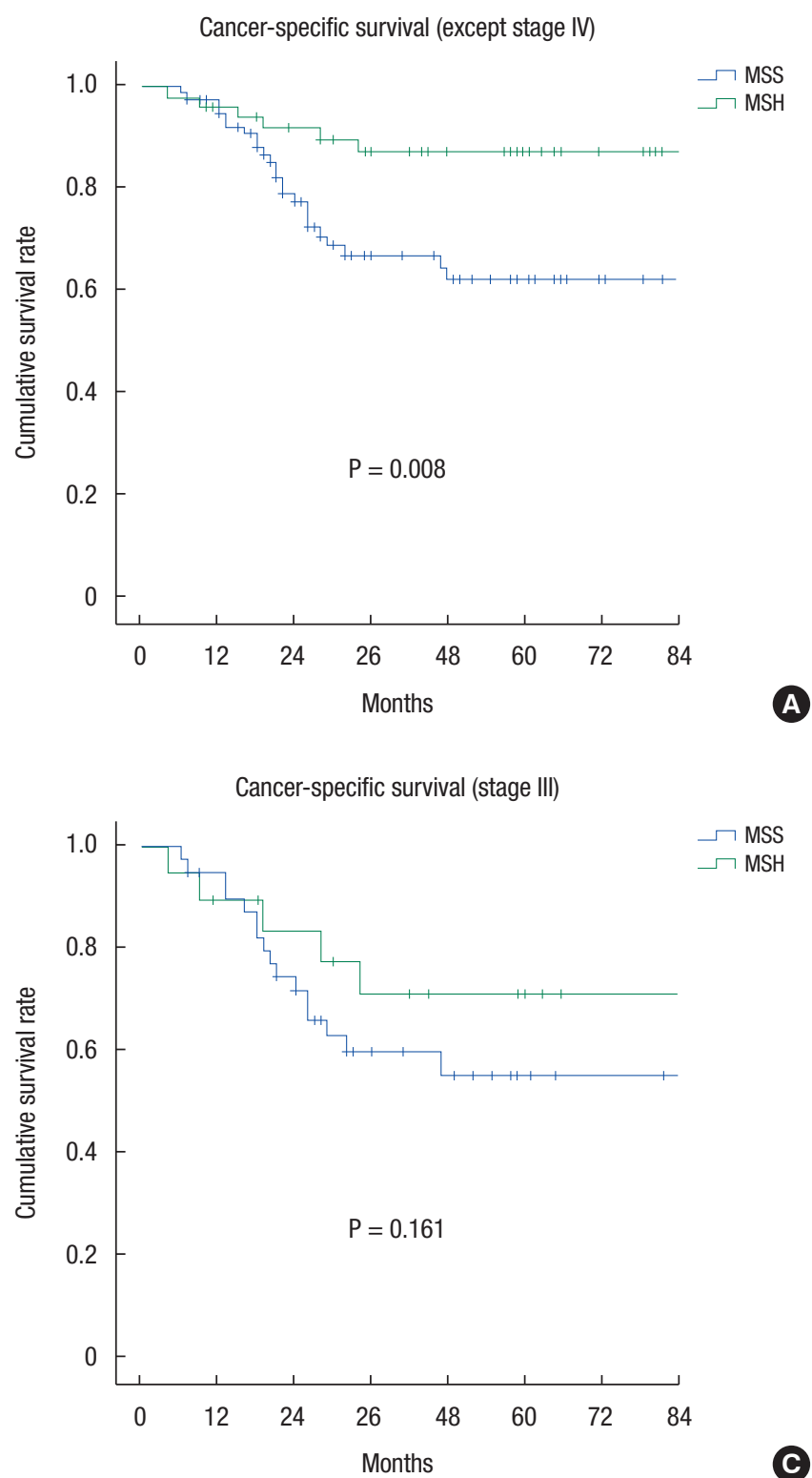

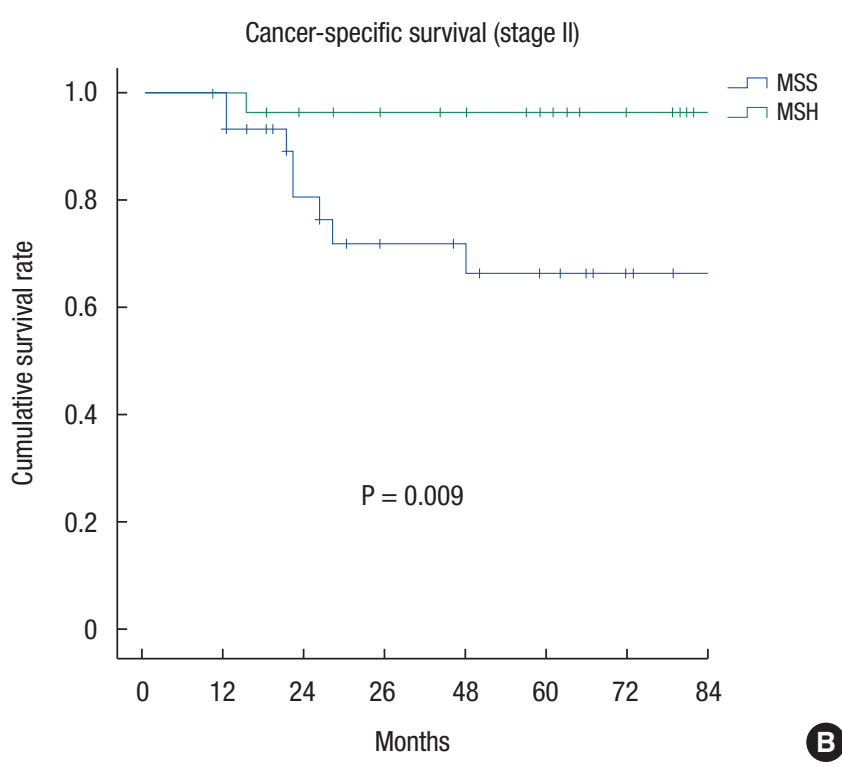

B

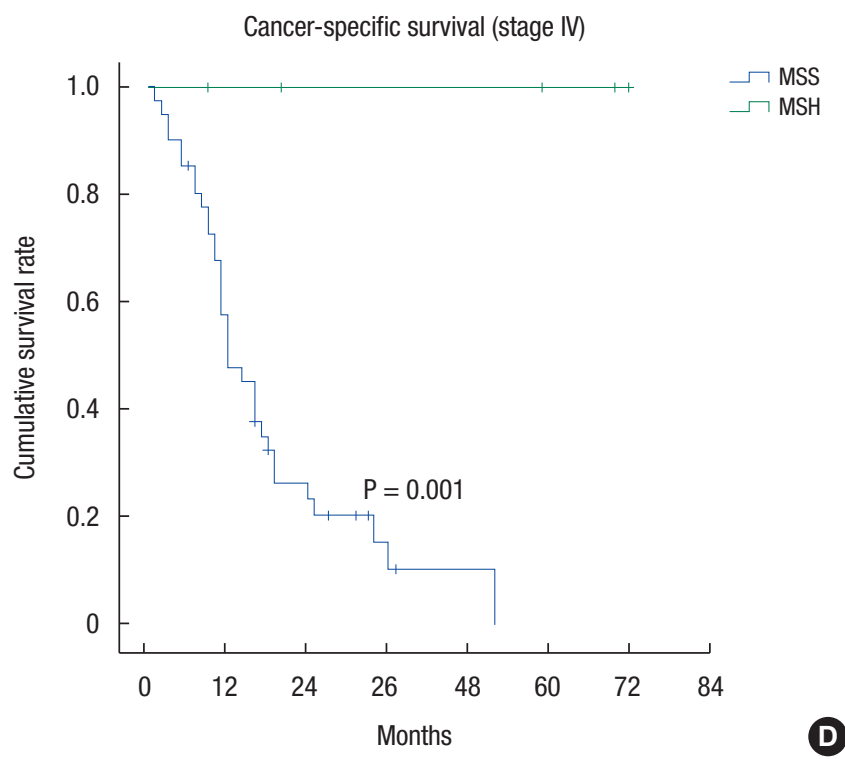

Fig. 2. Kaplan-Meier survival curves showing the 5-year cancer-specific survival according to MSI- status (substage). (A) All except stage IV, (B) stage II, (C) stage III, and (D) Stage IV.

ratio, 4.340, 95\% confidence interval, 1.680-11.213) (Table 3).

\section{DISCUSSION}

The degree of differentiation, as well as some histologic morphologies, of colorectal adenocarcinomas has been considered to reflect the grade of malignancy. CRCs presenting with MUCs, SRCs, or PDCs have been noted to have poor prognosis and to present with more advanced disease stage compared with CRCs with well- or moderately differentiated cells [11]. However, the prognoses for patients with MUC/SRC/PDC CRCs are not uni- form $[13,14]$. This may result from the small number of patients with, and the different molecular features for, colorectal carcinogenesis in patients with MUC/SRC/PDC CRCs [15]. The occurrence of high-MSI in patients with $\mathrm{CRC}$ has been noted to be associated with a favorable prognosis compared with the occurrence of MSS in patients with CRC. Interestingly, patients with high-MSI cancers tend to show MUCs/SRCs/PDCs, which have bad clinical or pathologic features [11]. Therefore, MSI status may be an important factor for giving a prognosis for patients with CRCs presenting with MUCs/SRCs/PDCs. In this study, we found that high-MSI CRC with the presence of MUCs/SRCs/ 
Table 3. Risk factors influencing cancer specific survival as identified by using a Cox-regression analysis (univariate and multivariate analyses)

\begin{tabular}{lcr}
\hline Univariate analysis & Cancer-specific survival & P-value \\
\hline Male sex & $1.297(0.782-2.152)$ & 0.314 \\
Age $>55 \mathrm{yr}$ & $1.608(0.940-2.753)$ & 0.083 \\
CEA $>5 \mathrm{ng} / \mathrm{mL}$ & $1.892(1.140-3.139)$ & 0.014 \\
Left colon cancer & $1.734(1.052-2.858)$ & 0.031 \\
\hline Tumor size $>6 \mathrm{~cm}$ & $0.942(0.574-1.548)$ & 0.815 \\
Lymphatic invasion & $2.334(1.219-4.470)$ & 0.011 \\
Vascular invasion & $3.435(2.050-5.756)$ & $<0.001$ \\
Neural invasion & $3.560(2.109-6.009)$ & $<0.001$ \\
T4 invasion & $3.329(2.023-5.480)$ & $<0.001$ \\
N1 lymph-node metastasis & $3.578(1.158-8.098)$ & 0.002 \\
\hline N2 lymph-node metastasis & $7.254(2.738-11.237)$ & $<0.001$ \\
MSS/low & $6.298(2.709-14.638)$ & $<0.001$ \\
Synchronous distant metastasis & $6.327(3.828-10.459)$ & $<0.001$ \\
Multivariate analysis & & \\
(except distant metastasis) & & 0.075 \\
CEA > 5 ng/mL & $1.614(0.952-2.736)$ & 0.002 \\
\hline T4 invasion & $2.148(1.215-3.799)$ & 0.081 \\
\hline N1 lymph-node metastasis & $2.332(0.902-6.025)$ & 0.032 \\
\hline N2 lymph-node metastasis & $2.672(1.089-6.554)$ & 0.002 \\
\hline MSS/low & $4.340(1.680-11.213)$ & \\
\hline
\end{tabular}

Values are presented as hazard ratio (95\% confidence interval).

CEA, carcinoembryonic antigen; MSS, microsatellite stability.

PDCsI was an independent positive prognostic factor for CSS. In addition, high-MSI cancers appeared to have more favorable clinicopathologic features than MSS cancers [8].

High-MSI CRCs have been reported to present with a lower overall stage, and this was also confirmed in present study $[5,16$, 17]. CRCs presenting with MUCs/SRCs/PDCs are known to be histologic subtypes showing a high malignancy potential. In this study, about $90 \%$ of all MUC/SRC/PDC cancer tumors were T3 or T4 in invasion depth of the primary tumor regardless of MSI status. Especially, the rate of T4 tumors in MSS cancers was approximately $40 \%$, which is high compared with the values of about $15 \%-20 \%$ reported in the literature, as well as the values of $85 \%-90 \%$ for cancers with well- and moderately differentiated cells $[11,17]$. The incidence of stage II in patients with high-MSI cancers with MUCs/SRCs/PDCs was about 50\%, although that of patient with MSS cancer was approximately $26 \%$; the rate of stage II reported in the literature is generally $30 \%-35 \%[5,17]$. Moreover, 50\% with MSS cancer had four and more lymph-node metastases compared with about $12.5 \%$ with high-MSI cancers. The frequency of stage-IV MSS cancers at presentation (34.2\%) was higher than that of high-MSI cancers (about 9\%) $[2,18]$. This in- cidence for high-MSI cancers with MUCs/SRCs/PDCs was particularly low compared to the values of $15 \%-22 \%$ reported in the literature $[8,16]$. Therefore, patients with high-MSI CRCs with MUCs/SRCs/PDCs will present with a low TNM stage compared with patients with MSS CRCs. Although high-MSI cancer and MUC/SRC/PDC histologic subtypes are common in proximal colon compared with distal colon, the mechanism underlying this difference has still not been identified.

In the present study, the incidence of distant metastasis of highMSI CRCs presenting with MUCs/SRCs/PDCs after curative treatment was approximately $12 \%$, which was lower than that of MSS cancers [19]. The frequency of distant metastasis for MSS tumors was about 35\%, which is higher than the $20 \%-25 \%$ usually given in the literature [5]. This may highlight the invasiveness of most CRCs presenting MUCs/SRCs/PDCs without high-MSI. Hematogenous spread is a predominant metastasis pattern of CRC [5]. Hematogenous metastasis in MUC/SRC/PDC CRCs was under half of all systemic metastases, regardless of MSI status [19]. The low incidence of hematogenous metastases in our study is because of the high risks of metastatic systemic lymph-nodes in MSI cancers and of peritoneal metastasis in MSS cancers. Distant metastasis in MUC/SRC/PDC CRC without high-MSI appeared to be due to a tendency to spread to the peritoneum/ovaries, which we observed in about $50 \%$ of the patients with MSS tumors in our study $[17,18]$. This is comparable to previous findings that reported the rate of peritoneal metastasis in SRC, MUC or PDC patients to be over $50 \%$ [20]. In addition to $\mathrm{T}$ or $\mathrm{N}$ status, several tumor-related factors have been identified as adverse prognostic features. Vascular invasion represents a crucial step in the formation of micrometastases. A significantly low proportion of vascular invasion in high-MSI tumors was observed in our study [11], but the rate of neural invasion in patients with MUC/SRC/PDC CRC with MSS was high compared with other reports [18]. The high incidence of peritoneal metastasis and the relatively low incidence of hematogenous spread may be regarded as a characteristic systemic spread pattern for CRC presenting with MUCs/ SRCs/PDCs.

MUC/SRC/PDC CRCs, as a whole, have a more advanced pathological TNM stage and show worse patient survival than welland moderately differentiated cancers $[2,5]$. Some authors have reported that patients with MUC/SRC/PDC CRC located in the proximal colon have a better prognosis than those with MUC/ SRC/PDC CRC in a distal location $[14,21]$. The favorable prognosis of proximal MUC/SRC/PDC CRC might be partly attributed to the possible dominance of MSI cancers which show better prognosis than MSS cancers [8]. Compared with the reported incidence of about $7 \%$ for high-MSI in well- or moderately differentiated carcinomas, that for high-MSI in MUC/SRC/PDC cancers is reported to range from $20 \%$ to $40 \%$, and these results are consistent with the results of this study [11]. Most high-MSI tumors have been reported to be located proximal to the splenic flexure, which is consistent with our result of about $80 \%$ [15]. However, 
the frequency of high-MSI tumors in the left colon or rectal cancer was low, about $20 \%$. After adjusting for $\mathrm{T}$ and $\mathrm{N}$ status, year of diagnosis, primary tumor location, and vascular and neural invasion, high-MSI MUC/SRC/PDC CRC was independently associated with favorable outcomes compared with MSS CRC in present study. This was consistent with the result reported by Gryfe et al. [9] that high-MSI was a positive predictive factor in a multivariate analysis, including tumor stage. The precise explanation for the prognostic advantage due to MSI is still not clear, even though different immune responses and carcinogeneses have been advocated to be responsible for this clinical behavior [15].

We found that the 5-year CSS for patients with CRC presenting with MUCs/SRCs/PDCs was better for tumors showing MSI than it was for tumors showing MSS [9-11]. This finding was consistent with that for CRC patients who showed MUCs and PDCs, but not for patients having SRCs, the smallest number of patients in this study, who had the poorest survival. Especially, the 5-year CSS for patients with stage-II and -III high-MSI cancers was significantly better than that for patients with MSS cancer when compared stage by stage. On the other hand, only patients with stage-III high-MSI cancers showed better survival, but without significance [14]. Even though the number of patients in this series was not large enough to reach any definitive conclusion, the prognosis of patients with MUC/SRC/PDC CRC showing highMSI is significantly better than that for patients with CRCs showing MSS. Our finding of improved survival associated with MSI in patients with MUC/SRC/PDC CRCs is consistent with the findings of previous report [8]. This supports the recent WHO recommendation to grade mucinous carcinomas according to their MSI status [11]. The survival difference in patients with MUC/SRC/PDC CRCs according to MSI status suggests that the positive effect of MSI on survival is stronger than the negative effect of poor histologic features.

In conclusions, MUC/SRC/PDC CRCs have poor histopathologic features. However, MSI status is an independent prognostic factor for mucinous, signet-ring, and poorly differentiated CRCs and is associated with favorable outcomes. Despite the limitations of our retrospective review and our small sample size, our results demonstrated that patients with a colorectal adenocarcinoma presenting with MUCs, SRCs, and PDCs are better stratified by survival when the MSI status of the tumor is incorporated into the grading system.

\section{CONFLICT OF INTEREST}

No potential conflict of interest relevant to this article was reported.

\section{ACKNOWLEDGMENTS}

This work was supported by a 2013 Yeungnam University Research Grant.

\section{REFERENCES}

1. Thota R, Fang X, Subbiah S. Clinicopathological features and survival outcomes of primary signet ring cell and mucinous adenocarcinoma of colon: retrospective analysis of VACCR database. J Gastrointest Oncol 2014;5:18-24.

2. Hyngstrom JR, Hu CY, Xing Y, You YN, Feig BW, Skibber JM, et al. Clinicopathology and outcomes for mucinous and signet ring colorectal adenocarcinoma: analysis from the National Cancer Data Base. Ann Surg Oncol 2012;19:2814-21.

3. Kang H, O'Connell JB, Maggard MA, Sack J, Ko CY. A 10-year outcomes evaluation of mucinous and signet-ring cell carcinoma of the colon and rectum. Dis Colon Rectum 2005;48:1161-8.

4. Secco GB, Fardelli R, Campora E, Lapertosa G, Gentile R, Zoli S, et al. Primary mucinous adenocarcinomas and signet-ring cell carcinomas of colon and rectum. Oncology 1994;51:30-4.

5. Benedix F, Kube R, Meyer F, Schmidt U, Gastinger I, Lippert H, et al. Comparison of 17,641 patients with right- and left-sided colon cancer: differences in epidemiology, perioperative course, histology, and survival. Dis Colon Rectum 2010;53:57-64.

6. Bjerkeset T, Morild I, Mork S, Søreide O. Tumor characteristics in colorectal cancer and their relationship to treatment and prognosis. Dis Colon Rectum 1987;30:934-8.

7. Halvorsen TB, Seim E. Degree of differentiation in colorectal adenocarcinomas: a multivariate analysis of the influence on survival. J Clin Pathol 1988;41:532-7.

8. Raut CP, Pawlik TM, Rodriguez-Bigas MA. Clinicopathologic features in colorectal cancer patients with microsatellite instability. Mutat Res 2004;568:275-82.

9. Gryfe R, Kim H, Hsieh ET, Aronson MD, Holowaty EJ, Bull SB, et al. Tumor microsatellite instability and clinical outcome in young patients with colorectal cancer. N Engl J Med 2000;342:69-77.

10. Gafà R, Maestri I, Matteuzzi M, Santini A, Ferretti S, Cavazzini L, et al. Sporadic colorectal adenocarcinomas with high-frequency microsatellite instability. Cancer 2000;89:2025-37.

11. Rosty C, Williamson EJ, Clendenning M, Walters RJ, Win AK, Jenkins MA, et al. Should the grading of colorectal adenocarcinoma include microsatellite instability status? Hum Pathol 2014; 45:2077-84.

12. Boland CR, Thibodeau SN, Hamilton SR, Sidransky D, Eshleman JR, Burt RW, et al. A National Cancer Institute Workshop on Microsatellite Instability for cancer detection and familial predisposition: development of international criteria for the determination of microsatellite instability in colorectal cancer. Cancer Res 1998; 58:5248-57.

13. Xiao H, Yoon YS, Hong SM, Roh SA, Cho DH, Yu CS, et al. Poorly differentiated colorectal cancers: correlation of microsatellite instability with clinicopathologic features and survival. Am J Clin Pathol 2013;140:341-7.

14. Catalano V, Loupakis F, Graziano F, Bisonni R, Torresi U, Vincenzi B, et al. Prognosis of mucinous histology for patients with radically resected stage II and III colon cancer. Ann Oncol 2012; 


\section{3:135-41.}

15. Jass JR. Classification of colorectal cancer based on correlation of clinical, morphological and molecular features. Histopathology 2007;50:113-30.

16. Lin CC, Lai YL, Lin TC, Chen WS, Jiang JK, Yang SH, et al. Clinicopathologic features and prognostic analysis of MSI-high colon cancer. Int J Colorectal Dis 2012;27:277-86.

17. Mizushima T, Nomura M, Fujii M, Akamatsu H, Mizuno H, Tominaga $\mathrm{H}$, et al. Primary colorectal signet-ring cell carcinoma: clinicopathological features and postoperative survival. Surg Today 2010;40:234-8.

18. Chew MH, Yeo SA, Ng ZP, Lim KH, Koh PK, Ng KH, et al. Critical analysis of mucin and signet ring cell as prognostic factors in an Asian population of 2,764 sporadic colorectal cancers. Int J
Colorectal Dis 2010;25:1221-9.

19. Park JW, Chang HJ, Jung KH, Kim DY, Sohn DK, Han KS, et al. Clinicopathologic features of sporadic colorectal cancer with MLH1/MSH2 loss of expression - reduced likelihood of metastases. J Korean Soc Coloproctol 2008;24:175-83.

20. Pande R, Sunga A, Levea C, Wilding GE, Bshara W, Reid M, et al. Significance of signet-ring cells in patients with colorectal cancer. Dis Colon Rectum 2008;51:50-5.

21. Ishihara S, Watanabe T, Akahane T, Shimada R, Horiuchi A, Shibuya $\mathrm{H}$, et al. Tumor location is a prognostic factor in poorly differentiated adenocarcinoma, mucinous adenocarcinoma, and signet-ring cell carcinoma of the colon. Int J Colorectal Dis 2012; 27:371-9. 